\title{
Modeling the Epidemiological Landscape of Chronic Hepatitis C in a Sub-Saharan African Country: What Is Needed to Achieve Elimination by 2030 in Burundi?
}

\author{
Renovat Ntagirabiri ${ }^{*}$, Sarah Robbins ${ }^{2}$, Patrice Barasukana1, Belyse Munezero', \\ Martin Manirakiza1 \\ ${ }^{1}$ Kamenge University Hospital, Bujumbura, Burundi \\ ${ }^{2}$ Center for Disease Analysis Foundation, Lafayette, USA \\ Email: *ntagrenov@yahoo.fr
}

How to cite this paper: Ntagirabiri, R., Robbins, S., Barasukana, P., Munezero, B. and Manirakiza, M. (2018) Modeling the Epidemiological Landscape of Chronic Hepatitis $\mathrm{C}$ in a Sub-Saharan African Country: What Is Needed to Achieve Elimination by 2030 in Burundi? Open Journal of Gastroenterology, 8, 284-293.

https://doi.org/10.4236/ojgas.2018.88031

Received: June 21, 2018

Accepted: August 5, 2018

Published: August 8, 2018

Copyright $\odot 2018$ by authors and Scientific Research Publishing Inc. This work is licensed under the Creative Commons Attribution International License (CC BY 4.0).

http://creativecommons.org/licenses/by/4.0/

\begin{abstract}
Background: Chronic Hepatitis C virus (HCV) is an increasing threat to international public health, as it can have severe impacts on morbidity and mortality. Burundi has done little to address the hepatitis $\mathrm{C}$ burden in-country. This analysis aims to address the current epidemiological landscape of HCV in Burundi and identify efforts to achieve elimination by 2030. Methods: In order to estimate the chronic HCV population through 2030, an Excel-based Markov model was created; calibrated using historical data populated from a literature review and an in-country lead expert panel. Using this model, two scenarios were developed: Base 2017, continuing the current standard of care through 2030, and Elimination, where treatment and diagnosis were increased to achieve a $90 \%$ reduction in total infections by 2030 . Results: In 2017, there were estimated 392,000 viremic infections, with a viremic rate of 3.3\%. Under the Base 2017 scenario, the number of viremic infections is expected to decline by less than $15 \%$ by 2030 , while liver related morbidity and mortality are expected to increase, with an estimated 2100 deaths due to HCV by 2030 . Under the Elimination strategy, outcomes are estimated to significantly improve, with viremic infections declining by an estimated $90 \%$ by 2030 and HCV-related mortality expected to decrease by $85 \%$. More than 12,470 lives could be saved by achieving elimination by 2030. Conclusion: Achieving elimination of HCV in Burundi is reasonable with implementation of increased diagnosis and treatment efforts, including affordable programming to reduce liver-related morbidity and mortality.
\end{abstract}




\section{Keywords}

HCV, Epidemiology, Disease Burden, Modeling, Elimination

\section{Introduction}

Chronic Hepatitis C virus (HCV) is a blood-borne virus that impacts more than 71 million people worldwide [1]. It is an international public health threat due to its impact on liver related morbidity and mortality. Of those with chronic infection, $15 \%-20 \%$ will progress to liver cirrhosis within 20 years and in 2015, HCV was responsible for approximately 400,000 deaths, mostly from cirrhosis and hepatocellular carcinoma (HCC) [1].

Previously, treatment for HCV was restricted to Pegylated Interferon (PegIFN), a drug regimen requiring weeks of observed injections. More so, Peg-IFN was not suitable for all infected patients and often resulted in harsh side effects, such as nausea, fatigue, and other flu-like symptoms. Recent advances in medical research have led to the development of highly efficacious direct-acting antiviral therapy (DAAs). These pangenotypic drugs can, for the first time, produce sustained viral response rates (SVR) of close to $90 \%$ or more in patients, meaning a cure for the disease is plausible. However, access to HCV treatment remains limited, particularly in low and middle income countries where the current burden of Hepatitis $C$ is poorly understood and the healthcare system is strained [2].

In Burundi, a low income country in Eastern Africa, little has been done to address the growing hepatitis $\mathrm{C}$ burden in the country. Varying studies have reported rates from $6 \%$ [3] in healthcare workers to up to $27.1 \%$ in an adult patient population [4]. In a national HIV sero-survey conducted in 2002, overall anti-HCV prevalence in the general population was found to be approximately $8 \%$ [5]. Considered a high endemic country, Burundi needs strategies grounded in representative and more recent data.

The goal of this analysis was to understand the current epidemiological landscape of chronic Hepatitis C in Burundi and identify what efforts are needed to achieve Elimination of the disease by 2030 .

\section{Methods}

\subsection{Country Disease Burden Model}

An Excel-based Markov model estimating the disease progression of HCV was created to estimate the chronic HCV infected population through 2030 [2]. A detailed description of the HCV disease progression model, grounded in the natural history of HCV infection, has been published previously [2] [6] [7] [8]. The background population, mortality and HCV epidemiology data of Burundi were used to calibrate the disease burden model and forecast the current and future disease burden of HCV. A literature review was conducted to identify all 
available peer reviewed and non-indexed sources regarding HCV prevalence, genotype distribution, diagnosis, and treatment in Burundi. All available data published between January 1, 1985 and June 2018 were identified through searches of PubMed and Embase. Studies in non-representative or high risk populations (patients, people who inject drugs (PWID), etc.) as well as studies published prior to 1985 were excluded. Non-indexed government reports, including those from the Ministry of Health and National Blood Transfusion Service and personal communication with country experts were included to supplement the available data. Articles were scored based on the population scope, the study sample size, and the year of analysis. The best scoring study was identified and this historical data were used to calibrate the disease burden model. The inputs and outputs were reviewed with a lead expert in country. A summary of these inputs can be found in Table 1.

A national HIV sero-survey conducted in Burundi in 2002 provided an anti-HCV prevalence rate of $8.2 \%$ in the general population [5]. As the model is used to forecast active infections, a viremic rate of $77.5 \%$ was applied, based on expert feedback of hospital based data. Thus, approximately 390,000 individuals $(316,500-2,678,100)$ were infected in 2002 , corresponding to a viremic prevalence rate of $5.4 \%$. Background population and mortality data were obtained by gender and five year age cohorts from the United Nations (UN) Population database [9]. As common in Eastern and Central Africa, the predominant genotype was G4 (92.7\%), followed by 5\% G1 and approximately 1.7\% G3 [10]. As no diagnosis registry exists in Burundi, it was assumed (based on expert input) that approximately $5 \%$ of the viremic population was diagnosed, and of that, about $10 \%$ are diagnosed each year. Thus, in 2010, a total of 19,500 patients have been diagnosed, of which approximately 1950 patients are newly diagnosed each year. Similarly, while no treatment registry exists, expert input was used to estimate 200 patients treated in 2015 with a combination of Peg-IFN and Ribavirin. This number was expanded to 600 patients in 2016 and doubled, to 1200 in 2017, due to the introduction of DAAs in the country. Lastly, liver transplantation is not available in Burundi, thus zero transplants have occurred in country [11].

Table 1. Key inputs of the Burundi HCV disease burden model.

\begin{tabular}{cccc}
\hline Input & Value & Year & Source \\
\hline Anti-HCV Prevalence & $8.2 \%$ & 2002 & Expert Input \\
Viremic Rate & $77.5 \%$ & 2016 & \\
Genotype Distribution & Glother, $0.0 \% \mathrm{G} 2,1.7 \%$ & 2014 & {$[10]$} \\
Previously Diagnosed (Viremic) & $\mathrm{G} 3,92.7 \% \mathrm{G} 4$ & & Expert Input \\
Annual Number Treated & 19,500 & 2010 & Expert input \\
Annual Liver Transplants & 1200 & 2017 & [11]
\end{tabular}

Table 1 summarizes the key inputs of the Burundi HCV disease burden model. Inputs are reported as value, year of estimate, and source. 
The Markov model used in this analysis is an open sourced model that has been published on extensively over the past 4 years. Modelers and epidemiologists in France, Greece, Australia, Egypt, Spain, and Portugal have independently reviewed the model and provided feedback for modifications and updates [12]. Since its inception in 2012, the model has undergone over 80 revisions and updates [12].

\subsection{Modeled Base Case and Elimination Scenario}

Two scenarios were developed in order to assess the current Hepatitis C disease burden and the strategy needed to achieve elimination by 2030: Base 2017 and Elimination (Table 2).

The Base 2017 scenario replicates the current standard of care, such that the current levels of diagnosis and treatment were to remain constant through 2030. In 2015, medical eligibility was set to $60 \%$ to represent patients still on Peg-IFN therapies, due to high rates of contraindications and frequent adverse events. However, starting in 2016, SVR was increased to $95 \%$ to capture patients starting on DAA therapy. Additionally, treatment age expanded from 20 - 74 to 15 - 79 years in 2016 and fibrosis restrictions were removed in 2017 making all patients eligible for treatment regardless of fibrosis score. The number of individuals treated increased to 600 individuals annually starting in 2016 and 1200 in 2017. However, as no screening programs are currently in place in Burundi, this number was expected to decline by $50 \%$ of 2016 estimates by 2030 . The number of newly diagnosed patients was kept constant.

A second scenario was developed in order to achieve Elimination of HCV by

Table 2. Inputs by scenario, 2016-2030.

\begin{tabular}{|c|c|c|c|c|c|c|c|}
\hline & 2016 & 2017 & 2018 & 2019 & 2020 & 2021 & $\geq 2025$ \\
\hline \multicolumn{8}{|l|}{ Treated } \\
\hline Base 2017 & 600 & 1200 & 1100 & 1100 & 300 & 300 & 300 \\
\hline Elimination & 600 & 1200 & 1500 & 2000 & 10,000 & 22,000 & 35,000 \\
\hline \multicolumn{8}{|c|}{ Newly Diagnosed } \\
\hline Base 2017 & 1900 & 1900 & 1900 & 1900 & 1900 & 1900 & 1900 \\
\hline Elimination & 1900 & 1900 & 5000 & 8000 & 15,000 & 25,000 & 34,000 \\
\hline \multicolumn{8}{|l|}{ Fibrotic Stage } \\
\hline Base 2017 & $\geq \mathrm{F} 3$ & $\geq \mathrm{F} 0$ & $\geq \mathrm{F} 0$ & $\geq \mathrm{F} 0$ & $\geq \mathrm{F} 0$ & $\geq \mathrm{F} 0$ & $\geq \mathrm{F} 0$ \\
\hline Elimination & $\geq \mathrm{F} 3$ & $\geq \mathrm{F} 0$ & $\geq \mathrm{F} 0$ & $\geq \mathrm{F} 0$ & $\geq \mathrm{F} 0$ & $\geq \mathrm{F} 0$ & $\geq \mathrm{F} 0$ \\
\hline \multicolumn{8}{|l|}{ Treated Age } \\
\hline Base 2017 & $15-79$ & $15-79$ & $15-79$ & $15-79$ & $15-79$ & $15-79$ & $15-79$ \\
\hline Elimination & $15-79$ & $15-79$ & $15-79$ & $15-79$ & $15-79$ & $15-79$ & $15-79$ \\
\hline \multicolumn{8}{|l|}{ SVR } \\
\hline Base 2017 & $95 \%$ & $95 \%$ & $95 \%$ & $95 \%$ & $95 \%$ & $95 \%$ & $95 \%$ \\
\hline Elimination & $95 \%$ & $95 \%$ & $95 \%$ & $95 \%$ & $95 \%$ & $95 \%$ & $95 \%$ \\
\hline
\end{tabular}

Table 2 summarizes the annual inputs of the Base 2017 and Elimination Scenario. The number treated, newly diagnosed, as well as Fibrotic Stage, Treated Age, and SVR Rates are reported annually through 2025. 
2030. For the purposes of this modelling study, elimination was defined as a $90 \%$ reduction in total viremic infections. The model was used to estimate what increases in diagnosis and treatment were necessary to achieve this overall reduction. Medical eligibility remained the same as in the Base 2017 scenario (patients $\geq$ F0, 15 - 79 years of age, and 95\% SVR), while treatment and diagnosis were increased.

\section{Results}

Through the literature search, 20 studies published between Jan 1, 1985, and June, 2018 were identified through PubMed $(n=7)$ and Embase $(n=13)$ searches. After removing duplicates $(n=4), 16$ studies were selected for review and inclusion in the final analysis (Figure 1).

There was an estimated 392,000 (95\% CI: 144,800 - 590,900) viremic infections in 2017, corresponding to a viremic prevalence of 3.3\% (95\% CI: $1.2 \%$ 5.0\%). Almost half of all infections were found in those aged 20 - 49 (Figure 2), with a bimodal age distribution. Due to the limited number of treatments occurring in country and aging of the infected population, the number of viremic infections is expected to decline to 347,000 by 2030 , less than a $15 \%$ decrease over the next fifteen years. More so, associated liver related morbidity and mortality is expected to increase under the current standard of care. In 2017, there were an estimated 3000 decompensated cirrhosis cases and 1300 hepatocellular carcinoma

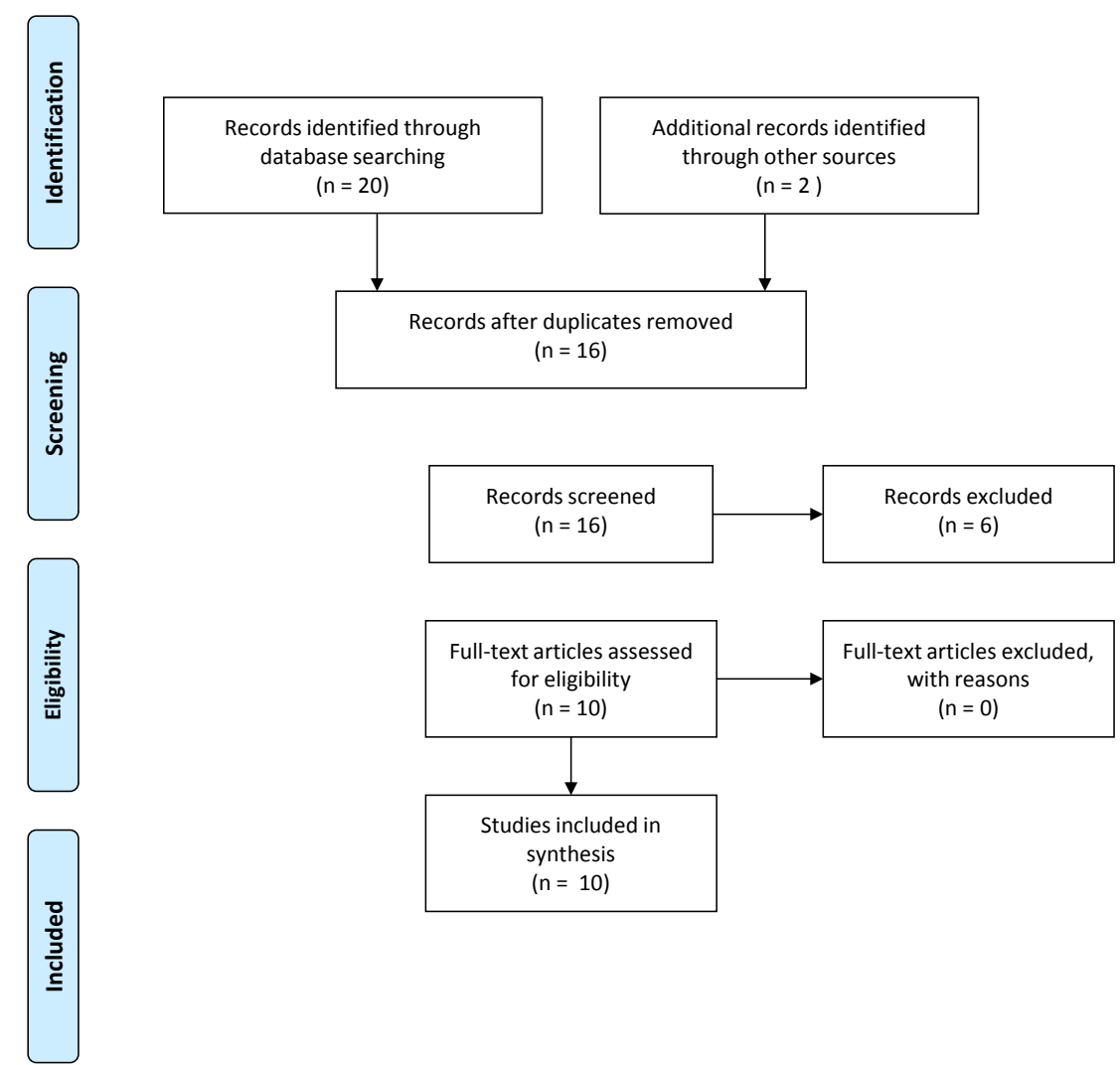

Figure 1. Flow chart of included studies. 
HCV Infected Population by Age Group - Burundi, 2017

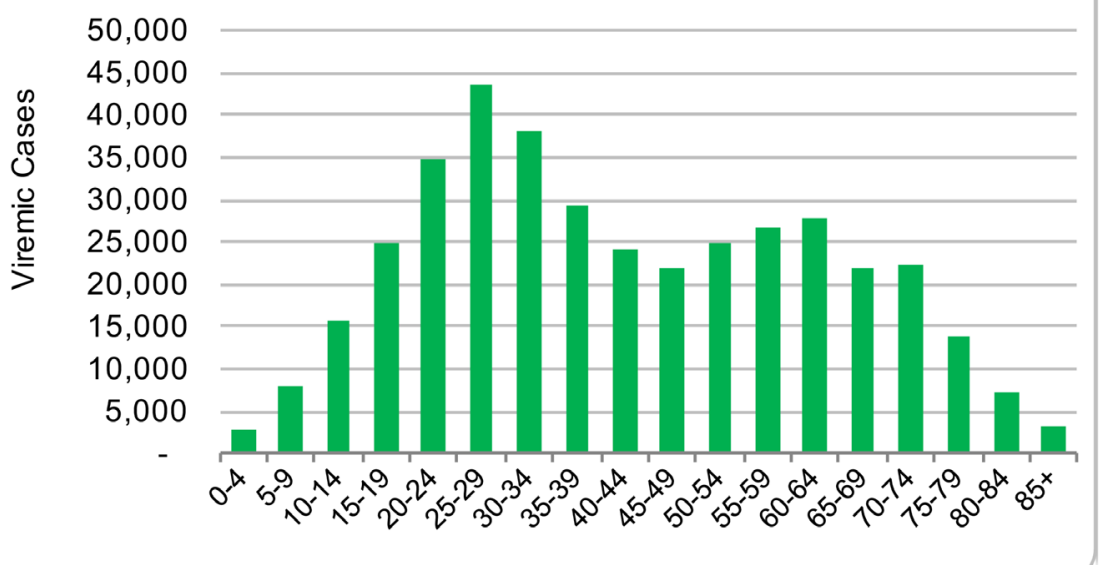

Figure 2. Estimated viremic HCV infected population by age in 2017. The age distribution of cases shows a bimodal curve in Burundi, suggesting an older wave of infected individuals (in those aged 50 - 64) and a newer insurgence of infections, particularly in those aged 20 - 34 .

cases. These numbers are forecasted to increase by $45 \%$ by 2030 , to 4400 and 1900 cases, respectively. Additionally, liver related mortality is expected to increase by $40 \%$ by the year 2030 . There will be an estimated 2100 deaths due to $\mathrm{HCV}$ if no treatment or disease management strategy is put into place.

To achieve Elimination of HCV in Burundi by 2030, drastic increases in both diagnosis and treatment are necessary. The number of treated patients will need to increase to 35,000 patients annually starting in 2025 and the number of newly diagnosed also needs to increase to 34,000 patients annually by the same year in order to achieve a $90 \%$ reduction in total viremic infections by 2030 .

Under the Elimination strategy, outcomes are expected to improve significantly (Figure 3 ). Total viremic infections are expected to decline by $90 \%$, to less than 39,000 infections by 2030. Liver related mortality and late stage liver disease is forecasted to decline considerably as well. Total decompensated cirrhosis cases are expected to decline by $85 \%$, from 3000 in 2016 to 390 in 2030. HCC cases will decline by the same proportion, from 1300 to 200 in 2030. Finally, HCVrelated mortality will decrease by $85 \%$ to 220 deaths in 2030 . Under this scenario, 6900 incident cases of HCC and more than 5400 incident cases of decompensated cirrhosis could be averted. More than 12,470 lives could be saved by achieving elimination by 2030 .

\section{Discussion}

Recent global and regional estimates have shown a wide variance of viremic infections across the African continent. This is most likely due to the sensitivity and specificity of the types of serological tests used and the fluctuating modes of transmission across the continent [13]. A recent World Health Organization 


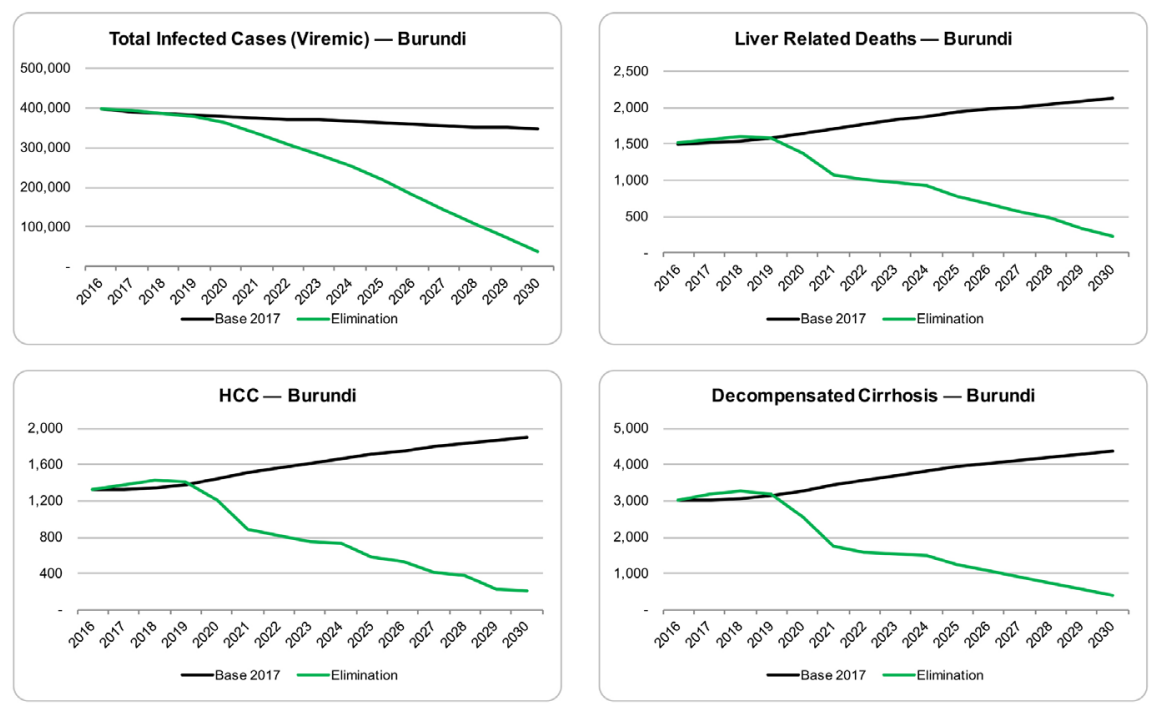

Figure 3. Forecasted HCV-related morbidity and mortality by intervention strategy, 2016 through 2030. The Base 2017 strategy showcases the outcomes of the current standard of care. The Elimination strategy (in green) results in an $85 \%-90 \%$ decrease in HCV-related infections, liver related morbidity and mortality.

(WHO) report noted that in the African region, there were an estimated 31 per 100,000 new incident HCV infections in 2015 [1]. While the Eastern Mediterranean Region and European Regions experience almost double the number of incident cases, 62.5 and 61.8, respectively, the African region experiences a younger infected population. This may mean a new wave of infections in the continent, especially as limited prevention and treatment programs exist. Sub-Saharan Africa, specifically, was found to have more than 30 times new infections than cures in 2016 [14]. More so, the African region was estimated to have one of the highest prevalence rates, with an estimated $1 \%(0.7 \%-1.6 \%)$ or approximately 11 million $(7,000,000-16,000,000)$ cases in 2015 . Overall total infections are expected to decline minimally over the next fifteen years throughout Africa, due to the increase in mortality. According to the WHO report, the largest burden of mortality associated with hepatitis of any WHO region is in Africa, with 13.7 deaths per 100,000 in 2015. In Sub-Saharan Africa, the number of estimated deaths due to cirrhosis almost doubled from 53,000 in 1980 to 103,000 in 2010 [13].

These outcomes, though dire, are expected, as access to HCV diagnosis and treatment remain quite limited across the African continent. According to the WHO, approximately $6 \%$ of the infected population was diagnosed in 2015, while less than $1 \%$ was on treatment [1]. This is echoed in Burundi, where treatment and prevention efforts have been restricted. This analysis estimates that of the infected population in $2016(396,000)$, only 5\% have been diagnosed and less than $1 \%$ were on treatment. If Burundi is to achieve elimination, it is clear that drastic efforts to link patients to care and initiate treatment are needed; however, some strides have recently been made in the country. In 2017, treatment restrictions were removed, thus making all patients eligible for treatment. 
This could potentially open access and increase the number screened and diagnosed in the country, the largest bottleneck to achieving elimination. Targeted screening strategies could be implemented throughout Burundi in order to improve this cascade of care. Recommendations from other regions throughout Southern Africa have suggested strategies focusing on key risk groups or modes of transmission [13]. Birth cohort screening in those aged $20-49$ or $50-64$ in Burundi may be justified in order to diagnose the largest portion of the infected population. More so, risk factor screening, such as screening anybody who has ever received a blood transfusion or medical procedure prior to blood screening, could be useful [13]. This is particularly true in Burundi, where the main modes of transmission were found to be nosocomial and related to traditional practices, as reflected in the bimodal age distribution of infections. Screening programs targeting those with tattoos, including traditional practice markings, could be included as well [13].

More so, there is a need to improve the education and awareness of general practitioners and other care providers when screening and diagnosing hepatitis $\mathrm{C}$ patients throughout Burundi. General practitioners (GPs) can contribute significantly to improving linkage to care; however, strategic programs of health education and awareness for both professional and risk groups are warranted [15]. According to a surveillance report in Europe, in 2012, only $21 \%$ of hepatitis $\mathrm{C}$ cases were diagnosed in general practice [16]. Making GPs aware of risk factors may efficiently improve case identification [15] and diagnosed and treated [17]. In Burundi, where standard screening practices are lacking, educated GPs can help identify patients and improve treatment adherence. This, coupled with increased sensitization of the general population, can enhance testing uptake [18], both of which are necessary steps if Burundi is to achieve elimination by 2030.

This is the first modelling analysis to forecast the current and future epidemiology of Hepatitis C virus infection in Burundi and estimate what is necessary in order to achieve elimination of the disease over the next fifteen years. However, this modelling work is dependent upon quality and availability of data, which is limited throughout Africa. Other reports have noted the scarcity of reliable prevalence data and population based studies, as well as the lack of estimates of the number diagnosed and treated annually in country [13] [14]. To capture these uncertainties, all inputs were validated by experts and prevalence was estimated with a 95\% uncertainty interval. However, in Burundi and across the continent, more recent nationally representative surveys are warranted. Additionally, the adoption of a national registry to track notifications and identify the treated population could be necessary if Burundi is to achieve elimination in the next fifteen years. This modelling study did not consider the costs associated with treatment and diagnosis as it was outside the scope of the original analysis. While the cost of treatment, particularly in low-income countries, has been reduced in recent years, further negotiations at the country level will be needed in 
order to increase access to diagnostics and treatment for all. A further evaluation examining the cost-analysis and affordability of such elimination programming would be required as the first step towards developing an elimination plan.

\section{Conclusion}

The goal of achieving elimination of HCV in Burundi is plausible, if efforts to substantially increase the diagnosed and treated population are implemented. Efforts to expand affordable programming and reduce the expected increase in late stage liver related morbidity and mortality could be assessed.

\section{Acknowledgements}

This study was supported through the CDA Foundation's Polaris Observatory (www.cdafound.org). Authors wish to thank Mindi DePaola for her feedback and formatting assistance.

\section{Competing Interests}

The authors declare no conflict of interest.

\section{Authors' Contributions}

SR conducted the literature review and RN provided data. All authors contributed to the analysis, prepared the draft and final manuscript. The authors approve of the final version.

\section{References}

[1] World Health Organization (2017) Global Hepatitis Report 2017. Geneva.

[2] Blach, S., et al. (2017) Global Prevalence and Genotype Distribution of Hepatitis C Virus Infection in 2015: A Modelling Study. The Lancet Gastroenterology \& Hepatology, 2, 161-176. https://doi.org/10.1016/S2468-1253(16)30181-9

[3] Le Pont, F., et al. (2003) Assessment of Occupational Exposure to Human Immunodeficiency Virus and Hepatitis C Virus in a Referral Hospital in Burundi, Central Africa. Infection Control \& Hospital Epidemiology, 24, 717-718. https://doi.org/10.1086/502908

[4] Aubry, P., et al. (1997) Seroprevalence of Hepatitis E Virus in an Adult Urban Population from Burundi. American Journal of Tropical Medicine and Hygiene, 57, 272-273. https://doi.org/10.4269/ajtmh.1997.57.272

[5] Ntagirabiri, R., et al. (2014) Prevalence of Hepatitis C Virus in Burundi: A Nationwide Survey. Journal Africain d Hepato-Gastroenterologie, 8, 25-28. https://doi.org/10.1007/s12157-014-0511-6

[6] Razavi, H., et al. (2014) The Present and Future Disease Burden of Hepatitis C Virus (HCV) Infection with Today's Treatment Paradigm. Journal of Viral Hepatitis, 21, 34-59. https://doi.org/10.1111/jvh.12248

[7] Sibley, A., et al. (2015) The Present and Future Disease Burden of Hepatitis C Virus Infections with Today's Treatment Paradigm-Volume 3. Journal of Viral Hepatitis, 22, 21-41. https://doi.org/10.1111/jvh.12476

[8] Hatzakis, A., et al. (2015) The Present and Future Disease Burden of Hepatitis C 
Virus (HCV) Infections with Today's Treatment Paradigm-Volume 2. Journal of Viral Hepatitis, 22, 26-45. https://doi.org/10.1111/jvh.12351

[9] United Nations (2016) Department of Economic Social Affairs Population Division, World Population Prospects: The 2015 Revision. United Nations, New York.

[10] Ntagirabiri, R., et al. (2014) Genotypes and Subtypes of Hepatitis C Virus in Burundi: A Particularity in Sub-Saharan Africa. Pan African Medical Journal, 19, 69. https://doi.org/10.11604/pamj.2014.19.69.4580

[11] Transplantation, T.I.R.o.O.D.a. (2015) The International Registry on Organ Donation and Transplantation. http://www.irodat.org/?p=database\&c=HR\#data

[12] Razavi, H., et al. (2017) Hepatitis C Virus Prevalence and Level of Intervention Required to Achieve the WHO Targets for Elimination in the European Union by 2030: A Modelling Study. The Lancet Gastroenterology \& Hepatology, 2, 325-336.

[13] Sonderup, M.W., et al. (2017) Hepatitis C in Sub-Saharan Africa: The Current Status and Recommendations for Achieving Elimination by 2030. The Lancet Gastroenterology and Hepatology, 2, 910-919. https://doi.org/10.1016/S2468-1253(17)30249-2

[14] Hill, A.M., Nath, S. and Simmons, B. (2017) The Road to Elimination of Hepatitis C: Analysis of SVR versus New HCV Infections in 91 Countries. Hepatology, 66, 118A.

[15] Bechini, A., et al. (2016) The Role of the General Practitioner in the Screening and Clinical Management of Chronic Viral Hepatitis in Six EU Countries. Journal of Preventive Medicine and Hygiene, 57, E51-E60.

[16] Control, E.C.f.D.P.a. (2014) Hepatitis B and C Surveillance in Europe 2012. Stockholm.

[17] Ferrante, J.M., et al. (2008) Family Physicians' Knowledge and Screening of Chronic Hepatitis and Liver Cancer. Family Medicine, 40, 345-351.

[18] Harris, M., Ward, E. and Gore, C. (2016) Finding the Undiagnosed: A Qualitative Exploration of Hepatitis C Diagnosis Delay in the United Kingdom. Journal of Viral Hepatitis, 23, 479-486. https://doi.org/10.1111/jvh.12513 